\title{
The Current Situation and Strategy about Teenager-football Training in Sichuan Province
}

\author{
Hang $\mathrm{LI}^{1, \mathrm{a}}$ \\ ${ }^{1}$ Civil Aviation Flight University of China, Guanghan 618307,China \\ ahangli2013@yeah.net
}

\section{Keywords: Teenager Football Training System}

\begin{abstract}
The essay regards the current situation and strategy about teenager-football training in Sichuan province as the study object, and draws a conclusion which indicates the main reasons of low efficiency of football training are that lack of the ways of teenager-football training and the ignorance of the public football development. They come up some strategies like that developing school football training, strengthening the culture quality and professional quality in order to build up suited teenager-football training system for our province and they hope that they can make some contributions for football development in Sichuan.
\end{abstract}

\section{Introduction}

It is make sense to develop teenager-football training for football sustainable development. Teenager as our football back-up talent, they have responsibility to make progress for Chinese football and they can help China acquire sporting power. According to the successful development of soccer power in this world, we analyze that the common reason for successful football development is putting a high value on teenager-football training. For our country, we do not have perfect teenager-football training system, and we do not have regular rules for teenager-football competitions and personnel training.

Sichuan as a major province of economy and football, it has made some contributions to Chinese football development for holding football activities. With the development of Chinese sports revolution, teenager-football training in Sichuan began to make continuous development in the direction of the diversified ways. In this reform, we need to search for more scientific ways for teenager sports training system in order to enhance the teenager-football level.

\section{The current situation about teenager-football training in Sichuan}

Our teenager-football training has restricted by sports bureau for a long time. Since professional football was carried out, we have built football system which regards football club as main subject and assists football schools, amateur football club and teenager-football competitions.

This kind of system means there is no fee for our country and local government, no restriction for administrative management. And we have put teenager-football into market. Compared with other countries, we develop teenager football for the sake of training back-ups for professional football club and we neglect the importance of developing school football.

After 2000, the scale of the football league has expanded, each club all has their own back-ups, but liquidity has been reduced and athletes do not have good foundation which results in no star player. At the same time, there is no improvement for teenager-football development because they lack investment.

On account of long growth cycle for a professional football player, clubs cannot get maximum benefits from it, and the kind of behavior results in bad circulation for talents training system and 
bad performance in sports events. What's worse, it makes us lack good football player for a long time.

\section{The problems of teenager-football training in Sichuan}

The fundamental task for teenager-football training is to expand the number of teenager football players and train more qualified football players.

On account of sudden separation from traditional football development, we regard club as main part of football development, so the teenager football training has been stopped for the period. As a result, there will not be many qualified football players appeared in ten years. Many football schools have been broken down, the function of association of has been reduced and more attention has paid on grades, these problems all cause low quality of teenager football training in Sichuan.

\section{a. Lack of ways to training football talents}

At the moment, the main way to training football talents is up to professional football club in the world. While in Sichuan, we do similar to it. On the contrary, our development time is not enough and we do not have suited measures to train teenager players. At the same time, because of no improvement in football schools, no enough qualified teenager-football players can be choose from by professional clubs. Account of low efficiency of training teenager players, clubs pays more attention on main football players rather than teenager players.

\section{b. Football league matches separate from teenager-football training}

The depressing and low quality of football league matches and has connections with teenager-football training system. Pay more attention on matches results in lacking back-up talents. Teenager players have poor skills and they cannot be qualified with matches' rules, so it will delay the best time for matches. As a consequence, football league matches separate from teenager-football training.

\section{c. Ignorance development of public football}

Public football in some way indicates a province whole football level. While in Sichuan, public has less time to take part in football activities, and no thick atmosphere of studying football has been built up. In Italy, 80\% of teenagers can play football. And football even spread in rural areas. In our country, our province, football is not popular in most cases. And under the influence of this situation, parents have no passion for their kids to learn football skills.

\section{d. Poor circulation for talents training system}

At the moment, the teenager football players are most from professional clubs. The clubs belong to some companies, and teenager players belong to the clubs. And the clubs will make many restrictions for their teenager players to take part in activities. Many teenager players are trained by one club one coach, so their skills will be restricted. Moreover, poor circulation for talents training system stopped players to find more potential club. And no enough time for players to take part in football matches will result in losing best time show players' talent in league matches.

\section{Study for building up teenager- football training system in Sichuan}

a. Provide more ways to train football talents, expand the scales of football matches.

Besides clubs, football associations, football schools, we need to increase more amateur clubs with policy and law help. It is essential for us to provide more ways to train football talents. Depending on companies, taking advantage of social resource, with the help of the government policy will be the insurance of developing teenager-football development. Professional clubs should have more connections with amateur clubs and football schools. A t the same time, government should invest more money and encourage providing more ways to train football talents. Without support from ordinary people, the improvement of football could be impossible.

\section{b. Develop football school and make progress on athletes' culture quality}

According to football powers, pay more attention to develop school football is crucial. In order to develop school football, football needs to be built as a special course and put football as a 
traditional sport. In addition, build a communication center for football players from different places; hold all kinds of football activities for teenager players in different age, have more connection with local professional football clubs so that the goal to train qualified teenager players can be reached. Moreover, make some policy in order to encourage teenager training. Last but not least, enhance the school football training system; make people have passion into football, especially children and teenagers.

c. Pay more attention to develop players' culture quality and professional quality.

Pay more attention to develop players' culture quality means to make culture as a standard to measure players. Make plans needs to regard body training as main training in order not to make players have no skills for football. Take advantage of sports facility is foundation for body training. In order to develop players in all-round way, we need to make players have passion for football from an early age and pay attention for their long run. Not only training skillful players, but also train layers with culture quality. In order to achieve it, we need to strengthen the training system, to make skills and culture quality up to train teenager players in order not make players only have one talent.

\section{Reference}

[1] SunYi, Compared Chinese football-teenager training with Japanese, Journal of Beijing Sport University,2008, (9) 1173-1176

[2] LiuDexin, Comparative analysis of Chinese and foreign youth football training idea, China Education Innovation Herald,2

[3]GaoShangui, Reviewing the history of Chinese soccer backup talents cultivation and reflection, Journal of Hubei Sports Science

[4]ZhenMeng, New ideals for Chinese football development, Journal of Tianjin Sports Science [5]JiangMinlang, ZhouChiqian, Thinking about Chinese scientific football training., Shanxi normal university sports institute 